February 9. 1976 $\rightarrow$
PORTIOHS OE MIS_REFORT_ARE_ITLEGIBUE, It has been reproduced from the best available oopy to permit the broadest possible availablity.

\section{ZGS PROPOSAL}

Studies of $v$ Interactions in Deuterium

Subritted by:

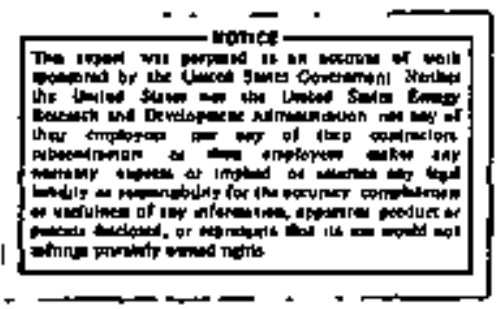

M. Derrick, T. Dombeck, L. G. Hyman, K. Jaeger, B. Musgrave, P. Schreiner, R. Singer, A. Snyder, and M. Szczekowski Argonne National Laboratory

5. J. Barish, A. Engler, G. Keyes, and 5. W. Kraeiner Carnegie-Mellon University

V. E. Barnes, D. D. Carmony, E. Fernandez, and A. F. Garfinkel Purdue University

I. Introduction

The study of neutrino interactions is currently one of the most exciting areas of experimental particle physics. Most important is the guestion of the form of the neutral curtent and the related question of the possible un: fication of the weak and electromagnetic interactions, Beyond these are the questions of whether presently accepted theory can properiy account for the various form factors in the charged-current reaclions.

The existence of the deuterium-filled 12 -foot bubble chamber in an intense neutrino beam has made it possible for physicists using them to más substantsal contributions to neutrino physics. The analysis of that data is nearing an end and we now propose to continue this worin us.ng tigher proton beam intensities, lower neutron backgrounds, and berter $\gamma$-ray detes. tion than was achieved in the previous experiment. Table $I$ summarizes thu 


\section{DISCLAIMER}

This report was prepared as an account of work sponsored by an agency of the United States Government. Neither the United States Government nor any agency Thereot, nor any of their employees, makes any warranty, express or implied, or assumes any legal liability or responsibility for the accuracy, completeness, or usefulness of any information, apparatus, product, or process disclosed, or represents that its use would not infringe privately owned rights. Reference herein to any specific commercial product, process, or service by trade name, trademark, manufacturer, or otherwise does not necessarily constitute or imply its endorsement, recommendation, or favoring by the United States Government or any agency thereof. The views and opinions of authors expressed herein do not necessarlly state or reflect those of the United States Government or any agency thereof. 


\section{DISCLAIMER}

Portions of this document may be illegible in electronic image products. Images are produced from the best available original document. 
: everit numbers we found in the various runs of E234 for the most common final states $\mu^{-} \mathrm{p}^{+}{ }^{+}$and $\mu-\mathrm{p}$. As can be seen, the yield of evonts for the last run ( $D_{2}$ IV) that was done in December 1974 is substantially bigher than previous runs. This resulted from a combination of using the secont focussing horn and a greater extracted proton beam intensity from the zGS. Our cur: rent analysis status is that all the film has been double-scanned and measured, and we are doing the final physicist editing of the data from the December ' $74 \mathrm{D}_{2}$ run. We will have the final results based on the complete experiment available for the Aachen $y$ conference in June, and we plan to write a number of articles describing the results of the experiment during the summer of this year.

\section{I1. The Proposal}

We propose to take $10^{6}$ pictures of $\nu$ incident on the deuterium-filled We propose to take $10^{6}$ pictures of $\nu$ incident on the deuterium-fil
bubble chamber with $y$-ray converting plates placed as shown in Fig. 1 . The plates are each one-half of a convexsion length of tantalum thick.

The separation between the plates at the median plane is 12 inches. Our fiducial volume in 2234 was chosen to be $6^{\prime \prime}$ from the Scotchlite wall panels. We believe that we will be able to analyze the constrained $v$ events that occur anywhere in the bubble chamber even with the plates in place, and $s o$ the event loss coming from the presence of the plates will be quite small.

Most of the proposed pictures should be taken after Booster II is available, but we request a run of $200 \mathrm{~K}$ pictures ( $\sim 2$ weeks of operation) in CY 1976 before the booster is ready. This will ensure continuity in our physics program and allow us to test the improved shielding arrangement and the practicality of the plate arrangement. Assuming that the CY'76 run will be the equivalent of the December 174 run and that the booster will give an extracted proton beam intengity of $\mathrm{g} \cdot 10^{12}$ protong/pulse, then Table II summarizes the 


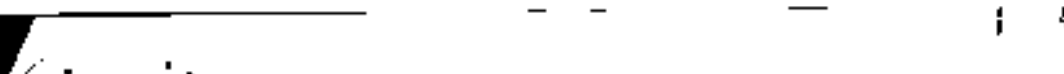

is event numbers expected in the new proposal and compares them to those of E234.

III. Neutrino Physics at the ZGS

The ZGS neutrino flux at the bubble chamber is shown in Fig. 2 . Since most of the $v$ intensity is below $1 \mathrm{GeV} / \mathrm{c}$, the phenomena of $\mathrm{V}-\mathrm{A}$ interfercnce is known to be large. This is very useful since it will not only allow a sensitive mea surement of the charged-current form factors, (1) but may enable us to measure the relative amounts of axial vector and vector contributions to single-pion production by the neutral current.

Another advantage of the ZGS flux shape is that one can study single* pion production below $1.5 \mathrm{GeV}$ with very little background from double and multi-pion production, as illustrated in Fig. 3. Single pion production, bccause of its simplicity, is an excellent reaction to explore the space and isotopic spin structure of the neutral current and test detailed models, such as Adler's, of charged interactions.

We currently know the neutrino flux to $\sim \pm 15 \%$ and believe that we can determine it even better. This allows us to measure absolute rates accurately and compare thern to theory.

\section{Neutrino Physics at Other Laboratories}

There is a substantial program of $v$ physice being carried out using the 7 -foot hydrogen bubble chamber at BNL and the 15 -foot bubble chamber at Fermilab (soon to be joined by the SPS at CERN). The spectrum at the 400 GeV machines has essentially no overlap with our experiment, and the physics aims are quite different. At $400 \mathrm{GeV}$ one stresses inclusive interactions and searches for new energy thresholds. Detailed studies of the neutral curzent interactions on single nucleons will be quite difficult. The BNI objectives are sim ilar to ours, but even there the energy regime is somewhat different - scaling 
"as the proton beam energy. This means that the ANL experiment is more suitable for form factor measurements, whereas BNL has more examples of multipion production. The overall event rate in the 7 -foot bubble chamber with $\nu$ in $D_{2}$ is about the same as we had in the December 174 run. With the booster, we anticipate a substantial rate advantage.

Spark chamber experiments have been done or are planned at BNL and CERN to specifically look at neutral current interactions. There has also been the big Gargamelle program that is now terminated at the CERN PS. These experiments have a substantial rate advantage over anything that can be done using a $\mathrm{H}_{2} / \mathrm{D}_{2}$ bubble chamber but, of course, the interactions are off a complex nucleus, and so the results have problems of interpretation.

\section{Neutral Current Pbysics}

\section{a. Single Pion Production by Neutral Currents}

Experiments to stucy neutral cur rents are difficult, and it probably will be some time before the experimental situation is clear. At this moment, the \$alam-Weinberg model provides an adequate description of the experimental facts, although even the form of the neutral current interaction is not known.

Three single pion production reactions can be studied:

$$
\begin{aligned}
& v_{\mathrm{n}}-v_{\mathrm{P}^{\pi}} \\
& v_{\mathrm{p}} \rightarrow v_{\mathrm{P}^{*}}{ }^{+} \\
& v_{\mathrm{p}} \rightarrow v_{\mathrm{n}}{ }^{+}
\end{aligned}
$$

Our group was the first to observe these reactions, (2) which allowed ug to confirm the existence of neutral cur rents.

Since our rate for the reaction $v_{\mathrm{p}}-v_{\mathrm{p}} \pi^{0}$ is higher than measured by other groups, we are interested fn regolving this discrepancy. By using the 
i plates to convert the $\gamma$-rays from $\pi^{\circ}$ decay, our detection efficiency for the $v{ }^{\circ}{ }^{\circ}$ final state will increase from $8 \%$ to about $30 \%$, and so the number of events we should expect in the new experiment is 67 , with one 6 converted either in the hydrogen or in the plates.

Adler ${ }^{(3)}$ bas shown how the study of these reactions will allow one to test the isospin structure and space-time siructure of the neutral current.

Or these channels, the reaction $v n-v p \pi^{-}$is special, because one gets complete information from these events such as the mass of the $p^{\pi}$ system, the $v$ energy, and momentum transfer. The new experiment will collect $\sim 120$ identified events. A principal background to this reaction comes from nn - np: ${ }^{-}$. In the present film, this background is important and in the early runs of E234 was comparable to the signal.

As we progressed in E234, we have improved the integrity of the shielding so that the rieutron background is now more than an order of magnitude lower than was the case for the first run. This is shown in Fig. 4, where we show the spectrum of recoil protons from the last $D_{2}$ exposure and compare it with earlier exposures. A second method of measuring the high momentum neutron flux comes from the number of one-constraint np $\mathrm{Pp}^{-}$fits found. As can be seen from Table II, there is only one such event in the last two deuterium exposureg. We bave completed a gurvey of the shielding as it now stands using a large scintillation counter as a neutron detector, and we have a detailed plan for shielding improvement that will further significantly reduce the neutron flux incident on the chamber. Consequently, the study of neutral current reactions will be much simpler in the new experiment, and our statistics will be further improved by our not having to make certain auts to reduce the background.

\section{b. Neutrino Elastic Scattering $v p-v p$}

The reaction $v p-v p$ is the most basic neutrino-nucleon interaction, 
'but it has yet to be observed. Upper limits on the cross section ratio $\sigma(\nu \mathrm{p}-\nu \mathrm{p}) / \sigma\left(\nu \mathrm{n}-\mu{ }^{-} \mathrm{p}\right)$ exist from CERN and Argonne. (4) There is great theoretical interest in elastic scattering and many calculations have been performed. For example, S. Ader ${ }^{(3)}$ has shown that for an isoscalar neutral current, one can relate the elastic cross section to that for single pion production:

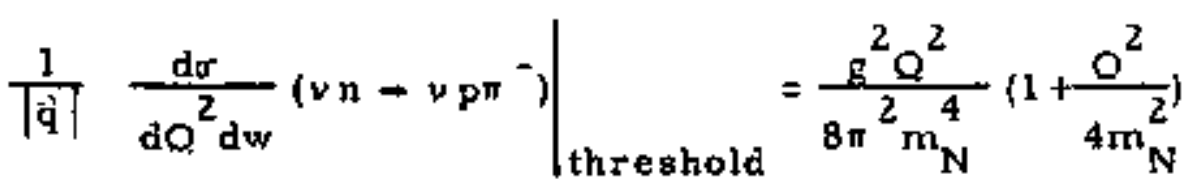

$$
\begin{aligned}
& x\left(J+\frac{Q^{2}}{2 m}\right)^{-2} \frac{d \sigma}{d C^{2}}(v p \rightarrow v p)
\end{aligned}
$$

Similar relations exist for the more general form of the neutral current interaction.

In the reaction $v \mathrm{p}-v \mathrm{p}$, one must go to relatively low proton momentum to achieve any sensitivity, but this is also the region having the highest background from np scatters. From the one-prong events measured in the April ' $73 \mathrm{D}_{2}$ run compared to December ' 74 , we find that shielding improvements have cut into the number of protons with momentum $>600 \mathrm{MeV} / \mathrm{c}$ by about a factor of $\approx 3.5$. In Fig. 5, we show the angular distribution of the protons from the $D_{2}$ IV run. The angle $\phi$ is the azimuthal angle about the neutrino beam direction. A value of $\phi=270^{\circ}$ means that the projection of the proton momentum pergendicular to the beam direction is vertically upward. Note the paucity of events in the range $200^{\circ} \leqslant \phi \leqslant 300^{\circ}$. The accumulation at $\phi-90^{\circ}$ is what one expects for neutrons coming downwards in the chamber and giving knock on protons, and we have been able to reproduce this kind of distribution with a Monte Carlo calculation.

In Fig. 6, we show a scatter plot of momentum versua angle relative to the $v$ beam direction. For $200^{\circ}<\phi<300^{\circ}$, we have made a box with $p \geqslant 600 \mathrm{MeV} / \mathrm{c}$ and $20^{\circ}<\theta_{\text {Bcat }}<60^{\circ}$. In this interval for $D_{2}$ (IV), there is 
one 'event. In the Weinberg theory with $\sin ^{2} \theta_{w}=0.35$, we expect 4.7 events in this range.

Although this is not final data and one mugt recognize that we arc quite sensitive to our scanning efficiency, it is quite striking that even withoul a background subtraction, there is a shoxtage of events expected for $\sin ^{2} \theta$ $\sim 0.35$. This is clearly a very important topic to pursue. The new exposure would yield over 350 elastic events over all $\phi$ with $P_{p} \geqslant 600 \mathrm{MeV} / \mathrm{c}$ and $20^{\circ} \leqslant \theta_{s} \leqslant 60^{\circ}$ (at $\sin ^{2} \theta_{w}=0.35$ ). The main problem is having a clear and complete understanding of the backgrounds.

\section{Charged-Current Physice}

\section{a. Quasi-Elastic Scattering $v n-\mu-p$}

Both the quasi-elastic $\mu{ }^{-} p$ and the $\mu{ }^{-} p^{+}{ }^{+}$final states can be selected by 3 -constraint kinematic fits and so the contamination in these event samples is small.

To describe quasi-elastic scattering theoretically, one wites down the most general V-A current and calculates the cross section in terms of six form factors. Using the usual simplications ${ }^{(5)}$ (no second class currents, pion dominance of the induced pseudoscalar form factor) seduces the problem to two form factors, the vector and axial vector. With the vector form factor being given by CVC, one parameterizes the axial form factor as

$$
F_{A}\left(Q^{2}\right)=\frac{1}{\left(1+Q^{2} / M_{A}^{2}\right)^{2}} \text {. }
$$

Based on our current experiment ${ }^{(1)}$ where we have done a complete analysis of a 396 event sample and get $\mathrm{M}_{A}=0.89+0.09$ GeV and assuming systematic errors do not begin to dominate, we anticipate the error on $M_{A}$ from 5000 events to be $\pm 0.025 \mathrm{GeV}$. We will have $\sim 250$ events with $\mathrm{O}^{2}>1 \mathrm{GeV}^{2}$, and so can probe to these large values of momentum transfer. 
With the large sample of events, we can also make a significant simuttaneous fit to both the vector and axial vector form factors. Assuming dipole forms, we expect $\Delta \mathrm{M}_{\mathrm{A}} \sim 0.07 \mathrm{GeV}$ and $\Delta \mathrm{M}_{\mathrm{V}} \sim 0.03 \mathrm{GeV}$ and thus will have a $4 \%$ check of the CVC prediction for $M_{V}$.

The elastic scattering events themselves check the flux normalization since the cross section at $Q^{2}=0$ is $1.98 \cdot 10^{-38} \mathrm{~cm}^{2} / \mathrm{GeV}$ (ignoring Pauli effects) independent of form factors. With a 5000 event sample, this will give a flux normalization error of $\neq 5 \%$.

\section{b. Single Pion Production Off Protons $v p-\mu-\mathrm{p}^{+}$}

Our measurements ${ }^{(6)}$ of the cross section and decay density matrix elements for the $\Delta^{++}$have ruled out a large number of calculations of this process. The Adler calculation (7) gurvived this check very well, although there are a number of form factors whose values are assigned in a somewhat arbitrary way, and have not been tested experimentally.

One can write the matrix element for $\Delta$ (1236) production using the notation in the review article by Llewellyn-Smith ${ }^{(5)}$ as

$$
\begin{aligned}
& M=\sqrt{\frac{3}{2}} G \bar{\psi} a\left\{\left[\frac{C_{3}^{V}}{m} \gamma_{\lambda}+\frac{C_{4}^{Y}}{m^{2}}\left(P_{\Delta}\right)_{\lambda}+\frac{C_{5}^{V}}{m^{2}}\left(P_{p}\right)_{\lambda}\right] \gamma_{5} F^{\lambda a}\right. \\
& +c_{6}^{V^{a} \gamma_{5}}+\left[\frac{C_{3}^{A}}{m} \gamma_{\lambda}+\frac{C_{4}^{A}}{m !}\left(P_{\Delta}\right)_{\lambda}\right] F^{\lambda a} \\
& \left.+C_{5}^{A} j^{\alpha}+\frac{C_{6}^{A}}{m^{2}}(q)^{a} q \cdot j\right\} U_{p}^{i}
\end{aligned}
$$

where $j_{a}$ is the lepton current, $F^{\lambda a}=q^{\lambda} j^{a}-q^{a} j^{\lambda}$, and the $C^{\prime}$ s are the four vector and four axial vector form factors. Time reversal invariance requires the form factors to be real, and the isotriplet current bypothesis allows the vector form factors to be related to those measured in electroproduction data. One finds $C_{5}^{V}=C_{6}^{V}=0, C_{4}^{V} \approx-\frac{M}{W} C_{3}^{V}$ and $C_{3}^{V}$ is well given by the dipole 
expression

$$
C_{3}^{v}=\frac{2.05}{\left(1+Q^{2} / 0.54\right)^{2}}
$$

One notes that the mass parameter is $0.54 \mathrm{GeV}^{2}$ instead of $0.71 \mathrm{GeV}^{2}$ as measured in elastic electron scattering.

The axial form factors could be determined with a sufficient number of events but at present, we must depend on model predictions. In particular, $C_{3}^{A}$ and $C_{4}^{A}$ are completely model dependent. $C_{6^{*}}^{A}$ the induced pseucloscalar, is taken as given by the pion pole term and only makes a amall contribution.

$C_{5}^{A}$ is determined by PCAC. Specifically at $Q^{2}=0$, we have.

$$
\frac{\mathrm{d} g}{\mathrm{dQ} Q^{2}}=\frac{\mathrm{G}^{2}}{2 \pi}\left(\frac{s-\mathrm{m}_{\Delta}^{2}}{s-\mathrm{m}_{\mathrm{p}}^{2}}\right)\left(\frac{\mathrm{m}_{\Delta}+\mathrm{m}_{\Delta} \mathrm{g}}{\mathrm{m}_{\Delta}}\right)\left(C_{5}^{A}\right)^{2} \text {. }
$$

so by measuring the cross section at $O^{2}=0$, one has a direct determination of $\mathrm{C}_{5}^{\mathrm{A}}(0)$. PCAC predicts $\mathrm{C}_{5}^{A}(0)=1,2$. Fig. 7 shows the low momentum transfer behavior of the cross section for the $\mu{ }^{*}{ }^{+}{ }^{+}{ }^{+}$final state together with PCAC prediction for $C_{5}^{A}$. This analysis is based on data up to the April ${ }^{+} 73$ run (see Table 1). We now have substantially more events. If one assumes $C_{3}^{A}=$ 0 and $C_{4}^{A}=-0.3$ as the Adler model requires, then the present data gives a check to PCAC to about $20 \%$. For a full check, one needs suffucient data to fix $C_{3}^{A}$ and $C_{4}^{A}$ experimentally, and we should be able to do this with results from the presently proposed experiment. The lines show how the cross section varies for $C_{3}^{A}$ and $C_{4}^{A}= \pm 1$ to give some idea of the sensitivity of the check to the values of the other form factors.

c. Single Pion Production Off Neutrons $\nu n-\mu^{-}{ }^{-} \pi^{\circ}, \mu^{-} n \pi^{+}$

By measuring and comparing results from the three single pion reactions $\nu p-\mu{ }^{-}{ }^{+}{ }^{+}, \nu n-\mu-p^{\circ}{ }^{\circ}, \mu{ }^{-}{ }^{+}{ }^{+}$, we have been able in E234 to separate the $\mathrm{T}=3 / 2$ and $\mathrm{T}=1 / 2 \pi \mathrm{N}$ amplitudes and place an upper limit on a possible $T \neq 2$ exchange amplitude. 
The events of single-pion production off neutrons, however, result in the 0 -constraint final states $\mu{ }^{-} \pi^{+}$and $\mu-\mathrm{pm}^{\circ}$. We have found it possible to separate the examples of sucb events from the non-neutrino background at the $10 \%$ level of accuracy. In particulár, the last runs (July and December '74) are quite free of background coming from neutron interactions and badron scatters. However, there are two sources of $v$-induced background that are difficult to correct for. For the $\mu{ }^{-} \mathrm{p}^{\circ}{ }^{0}$ final state, we find that the events satisfy the 3 -constraint kinematics of the quasi-elastic reaction $v n-\mu-p$ whenever the $\pi^{\circ}$ goes along the beam direction. For both the $\mu "{ }^{\circ} \pi^{\circ}$ and $\mu{ }^{-}{ }^{+} \pi^{+}$final states, we have not found it possible to remove background from the double pion production reactions such as $\mu{ }^{*} \pi^{\circ} \pi^{\circ}, \mu{ }^{-} \pi^{+} \pi^{\circ}$. To minimize the latter effect, we have only accepted events with $\mathrm{E}_{v}<1.5$ GeV, where the double pion production cross sections are relatively small (Fig. 3).

The $\pi$ Nmass spectrum from these two reactions extends above the $\Delta r$ egion as seen in Fig. 8. It would be interesting to see if indeed bigh mass $N^{*}$ 's are produced in $v$ interactions and with what cross section. To accomplish this, it is necessary to remove the $E_{\nu}=1.5 \mathrm{GeV}$ cut.

If one had good $Y$-ray detection, then many of these problems would be solved and so we propose to carry out the new experiment with $Y$-ray converting plates in the chamber. The calculated $\backslash$-ray momentum spectrum from single pion production is shown in Fig. 9. Unfortunately, the energies are low, which makes $\int$ detection and measurement rather difficult. The dashed line in Fig. 9 show the distribution of these 6 -rays which do hit the plates.

Final states having a $\pi^{\circ}$ will sign themselves about a third of the time . and for the particularly important sample of events with a forward $\pi{ }^{\circ}$, the detection efficiency will be much higher.

In the new experiment, we anticipate having about 500 events in each 


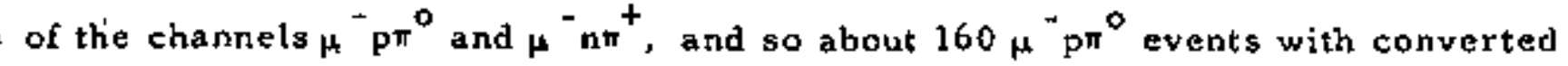
$\gamma$-rays. There will be also about 450 double pion production events in the final states $\mu^{-} \mathrm{p}^{\circ} \pi^{\circ}, \mu^{-} \mathrm{n} \pi^{+} \pi^{\circ}, \mu^{-} \mathrm{pm}^{+} \pi^{\circ}$ and $\mu{ }^{-} \mathrm{n \pi}^{+} \pi^{+}$.

With clean samples of several hundred $\mu^{-} \mathrm{pm}^{0}$ and $\mu^{-}{ }^{-} \pi^{+}$final states, one will be able to measure angular correlation in the $\Delta$ decay for these states as well as the $\mu{ }^{-} \mathrm{p}^{+}{ }^{+}$final state. We expect to refine the limit on a possible isotensor exchange to the $10 \%$ level and measure $A_{1} / A_{3}\left(A_{1,3}=T=\right.$ $1 / 2,3 / 2 \pi \mathrm{N}$ amplitude) to an accuracy of $5 \%$. With a total of aboul 3000 singie pion production events, we wial confront the Adler calculation with very good data.

d. Total Cross Sections for $\nu_{\mathrm{p}} \rightarrow \mu^{-}+\mathrm{x}^{++}$and $\nu \mathrm{n} \rightarrow \mu^{-}+\mathrm{X}^{+}$

We anticipate a total of about 9,000 events in the new experiment. 1,000 of which will have $\mathrm{E}_{v}>2 \mathrm{GeV}(700 \mathrm{un}$ and $300 \mathrm{vp}$ interactions). Since we can measure absolute cross sections, we can look at the energy variation of the total $v n$ and $v_{p}$ cross sections up to about $6 \mathrm{GeV}$ and measure the $\sigma(v n) / \sigma(v p)$ ratio. This is predicted by the quark parton model to be 1.56 in agreement with the present preliminary result ${ }^{(9)}$ of $1.40 \pm 0.31$ for $E_{v}>2 \mathrm{GeV}$. We will also look at the variation of this ratio as we make various kinematic cuts. The data will be compared to the expectations of the QPM in the scaling variables and compared to the electroproduction data.

- Although the number of events is not large, the energy span is also small so our data will provide precision low momentum points that may be compared to the high energy Fermilab data.

\section{e. Strange Particles}

E234 obtained the first data on strange particle production by neutrinos and, in particular, saw one example of associated production by a neutrad current. In the new experiment, we will collect $\sim 50$ examples of strange particle production and so begin to look at the characteristics of such events 
- in detail. Single kaon production seems to be comparable to as sociated production in the threshold region, which is interesting as it must resull from a $v$ interaction with the sea of qạ pairs in the nucleon. The two events found in $\mathrm{E} 234$ both have a pK mass just above threshold which is uncxpected. We do produce badronic states up to $2.5 \mathrm{GeV}$ in mass and so there is a chance to see evidence of charmed particles. In particular, if one believes the calculations of Shrock and Lee, we should see $\sim 10$ events of charuned particle production if the mass is $Z$ GeV. It is, of course, likely that there will be a rather complex spectroscopy of new particles. some of which could be within the mas sange of a low energy experiment such as we are proposing.

\section{Scanning and Measuring}

The scanning and measuring of the new flim will be done as it was for E234. The film will be double-scanned, mainly for the purpose of accurately measuring the scan efficiency for the one-prong topology. The scanning rate is presently 75 frames per hour. Thus, 27,000 hours are required for a double scan of $10^{6}$ frames. The scanners record not only neutrino-induced events, but also a large number of low energy hadron scatters. The rate of (hadron + neutrino) events is expected to be an event per 12.5 frames. If all of these events are measured, and if there are three measurement pas ses, a total of 14,000 hours are needed.

Thus, a total of 41,000 hours or 23 FTE scanner years are required to process the film. Of course, some scanners are also needed to do spacial editing. measure problem events using special techniques, and keep bookkeeping records, We, therefore, need 26 FTE acanner years of effort to do the scanning and measuring phase of the experiment. With three major groups involved, we anticipate completing the data analysis within two years. 


\section{References}

1. W. A. Mann et al, Phys. Rev. Letters 31, 844 (1973).

2. S. J. Barish et al., Phys, Rev. Letters 33, 448 (1973).

3. S. Adler, Phys, Rev, D12, 2644 (1975).

4. P. Schreiner, ANL/HEP 7436 (1974).

5. C. H. Llewellyn-Smith, Phys. Reports 3C, 261 (1973).

6. J. Campbell et al., Phys. Rev. Lotters 30, 335 (1973).

7. S. Adler, Annals of Physics 50, 189 (1968).

8. S. J. Barish et al., Phys. Rev. Lettérs 36, 179 (1976).

9. "An Inclusive Look at $v p$ and $v$ n Charged-Current Reactions Below $6 \mathrm{GeV}, " \mathrm{~s}$. J. Barish et al, , ANL-HEP-CP-75-38 given at SLAC Photon and Lepton" Conference, 1975.

10. S. J. Barish et a1., Phys. Rev. Letters 33, 1446 (1975).

11. "Second Class Currents," B. R. Holstein and S. B. Treiman, Princeton preprint (1976). 
Table I

Number of Events in the Two Most Abundant

Channels for Different Runs of E234

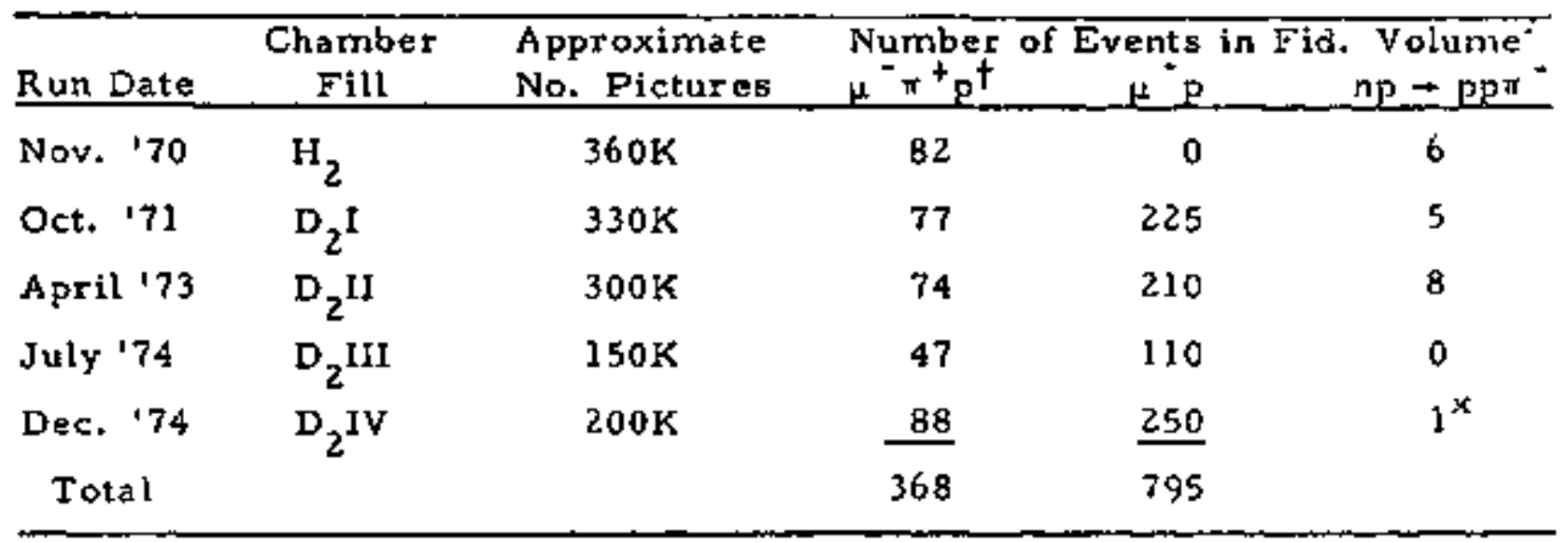

"Tnere are an additional $25-30 \%$ of events out side the fiducial volume that can be used for some distributions.

$t_{\text {In }} \mathrm{D}_{2}$ there is a $15 \%$ loss of events that have a fast neutron spectator.

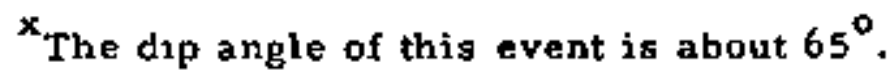


Table II

Number of Events Expected in the New Experiment as Compared to E234

\begin{tabular}{|c|c|c|}
\hline Reaction & $\begin{array}{l}\text { No. of Events } \\
\text { in } 2234\end{array}$ & $\begin{array}{l}\text { No. of Events Expected } \\
\text { in the Proposed Expt. }\end{array}$ \\
\hline \multicolumn{3}{|l|}{ Charged Currents } \\
\hline$v n-\mu^{*} p$ & 795 & 5000 \\
\hline$\nu p \rightarrow \mu^{-} \pi^{+} p$ & 368 & 1800 \\
\hline$\nu n \rightarrow \mu-\pi_{p}$ & 68 & 500 \\
\hline$\mu^{-} \pi^{+}{ }_{n} v^{-}$ & 82 & 600 \\
\hline$\nu_{n}-\mu^{-} \mathrm{p}^{+} \pi^{-}$ & 25 & 150 \\
\hline $\begin{array}{l}\nu p-\mu p^{-}{ }^{+}{ }_{2}^{\circ} \pi^{\circ} \\
\mu-n \pi^{+} \pi^{+} \ell \pi\end{array}$ & 73 & 450 \\
\hline$\geqslant 3 \pi$ Production & 31 & 200 \\
\hline Strange Particle Events & 8 & 50 \\
\hline $\begin{array}{l}\text { Total Charged-Current } \\
\text { Events }\end{array}$ & $\sim 1400$ & $\sim 9000$ \\
\hline \multicolumn{3}{|l|}{. Neutral Currents } \\
\hline$v_{p}-v_{p} \pi^{0}$ & 8 & 67 \\
\hline$v n \pi^{+}$ & 8 & 50 \\
\hline$v_{n}-\nu p \pi^{*}$ & 16 & 120 \\
\hline$v n-v K \Lambda$ & $\mathbf{l}$ & $\sim 5$ \\
\hline
\end{tabular}

"The number of events depends criticaliy on cuts needed to remove background. 


\section{Figure Captions}

Fig. 1 Suggested plate array for $Y$ conversion.

Fig. 2 v spectrum at ZGS.

Fig. 3 Excitation Iunction for quasi-elastic, $1 \pi, 2 \pi$, and $3 \pi$ production as measured in E234.

Fig. 4 (a) Spectrum of leaving protons from the December '74 run of E234. (b) Normalized ratio of one-prong rate on $\mathrm{H}_{2}$ exposure to April $173 \mathrm{D}_{2}$ run. (c) Normalized ratio of one-prongs, April ' 73 run to December ' 74 run.

Fig. 5 Distribution of 1-prong events around beam direction.

Fig. 6 Momentum versus angle for upward-going protons.

Fig. 7 d $7 / \mathrm{dQ}^{2}$ near $Q^{2}=0$ for $v p-\mu{ }^{-}{ }^{+}{ }^{+}$compared to the $P C A C$ prediction for $\mathrm{C}_{5}^{\mathrm{A}}$.

Fig. 8 in mass spectra from the $\mu{ }^{-} \mathrm{pm}^{+}, \mu^{-} \mathrm{pm}^{\circ}$, and $\mu^{-} \mathrm{n} \pi^{+}$rinal states.

Fig, 9 -ray momentum spectrum from the $\mu{ }^{-} p \pi^{\circ}$ final state. 


\section{APPENDIX}

\section{A Possible $\forall$ Exposure}

We have considered an antineutrino exposure in the chamber and have decided not to propose such an experiment at this time. An antineutrino experiment is significantly more difficult than one with neutrinos for a number ol reasons:

1. The event rate is about six times lower because of the lower $\bar{v}$ flux and the smaller $\dot{v}$ cross sections.

2. The high cross section channels have missing neutral particles

$$
\begin{aligned}
& \bar{v}_{\mathrm{P}} \rightarrow \mu_{\mathrm{n}} \\
& \bar{v}_{\mathrm{n}} \rightarrow \mu^{+}{ }_{\mathrm{n} \pi},
\end{aligned}
$$

so the backgrounds from events with an extra $\pi^{\circ}$ are difficult to bandle.

3. There tends to be more ambiguity in the choice of the muon for $v$ events than for $v$ events because of the net charge of the hadronic system.

4. There wilk be a substantial background of $v$-induced events in any $\bar{v}$ exposure which adds to the difficulty of the analysis.

Even so, there is much interesting physics to be done with an $\bar{v}$ experiment and such an exposure should certainly be made at some time with the ZGS $\nu$ facility. It is well known that a comparison of $v$ and $\dot{v}$ quasi-elastic scattering allows a very gensitive measure of $\mathrm{M}_{\mathrm{A}}$. There are allowed hyperon production channels with an $\bar{v}$ beam such as

$$
\dot{v}_{\mathrm{p}} \rightarrow p^{+} \mathrm{A}
$$

that have no background problems and on which essentially no information exists. Finally, as pointed out explicitly recently by Treiman and Holstein. a comparison of $\Delta$ production by $v$ and $\bar{v}$ beams provides a sensitive check of second class currents. 
After the booster is working, we anticipate coming to the commitice with a reçuest to run perhaps 100,000 pictures with an $v$ beam in order to experimentally study the difficulties of such an experiment and to follow this up with a full scale $\bar{v}$ experiment proposal. 


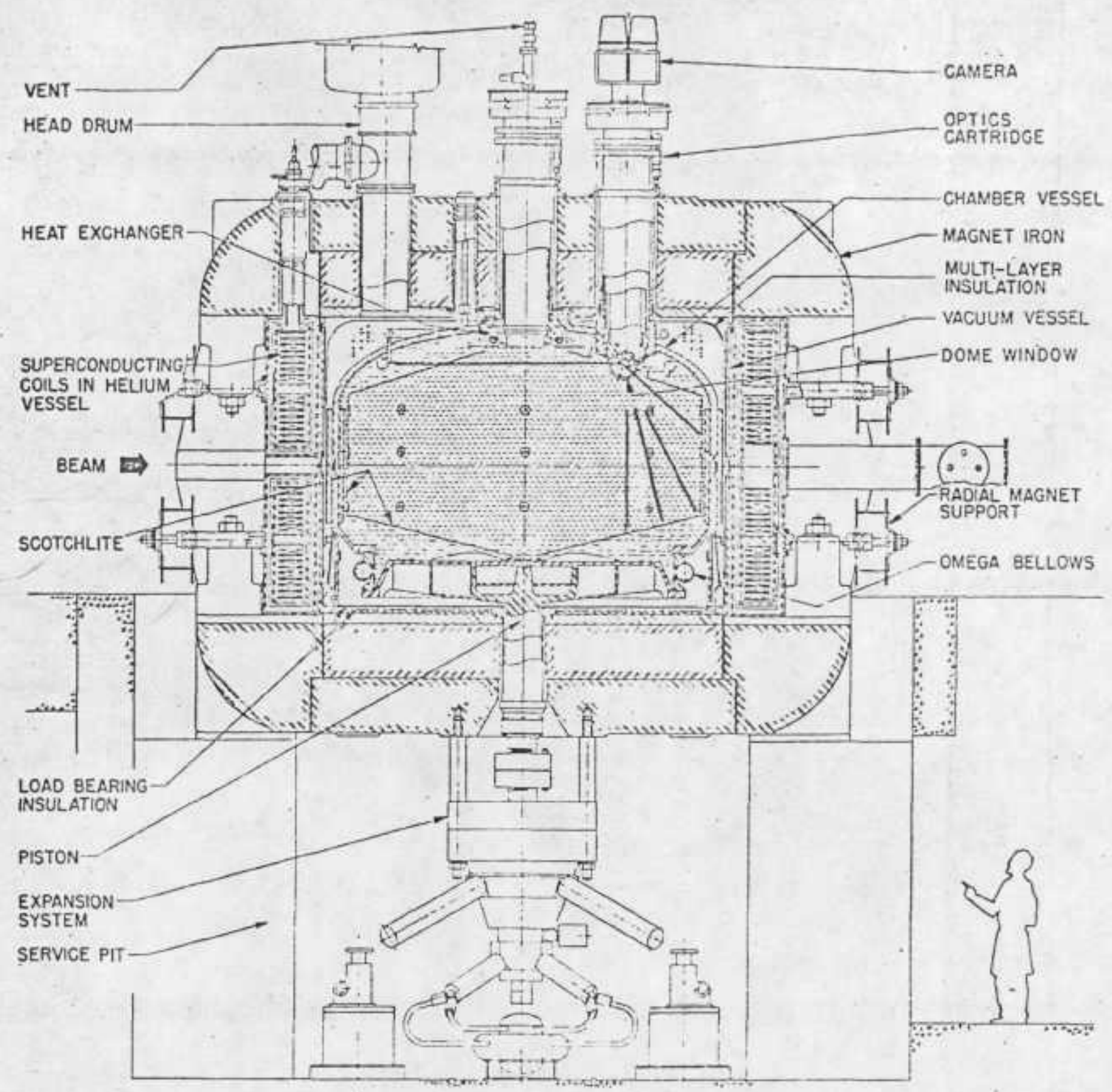

Fig, 1 


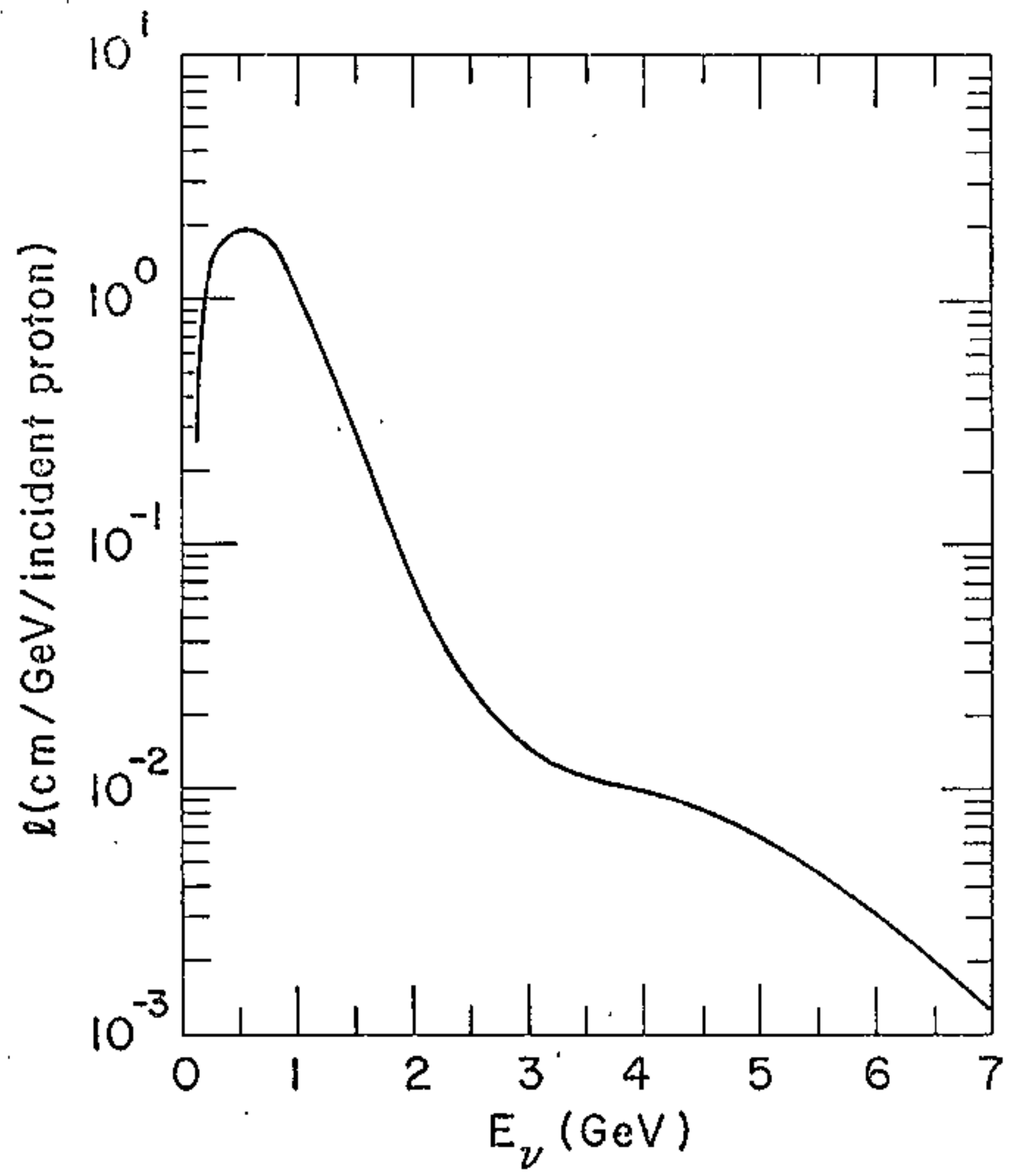

Fig. 2 


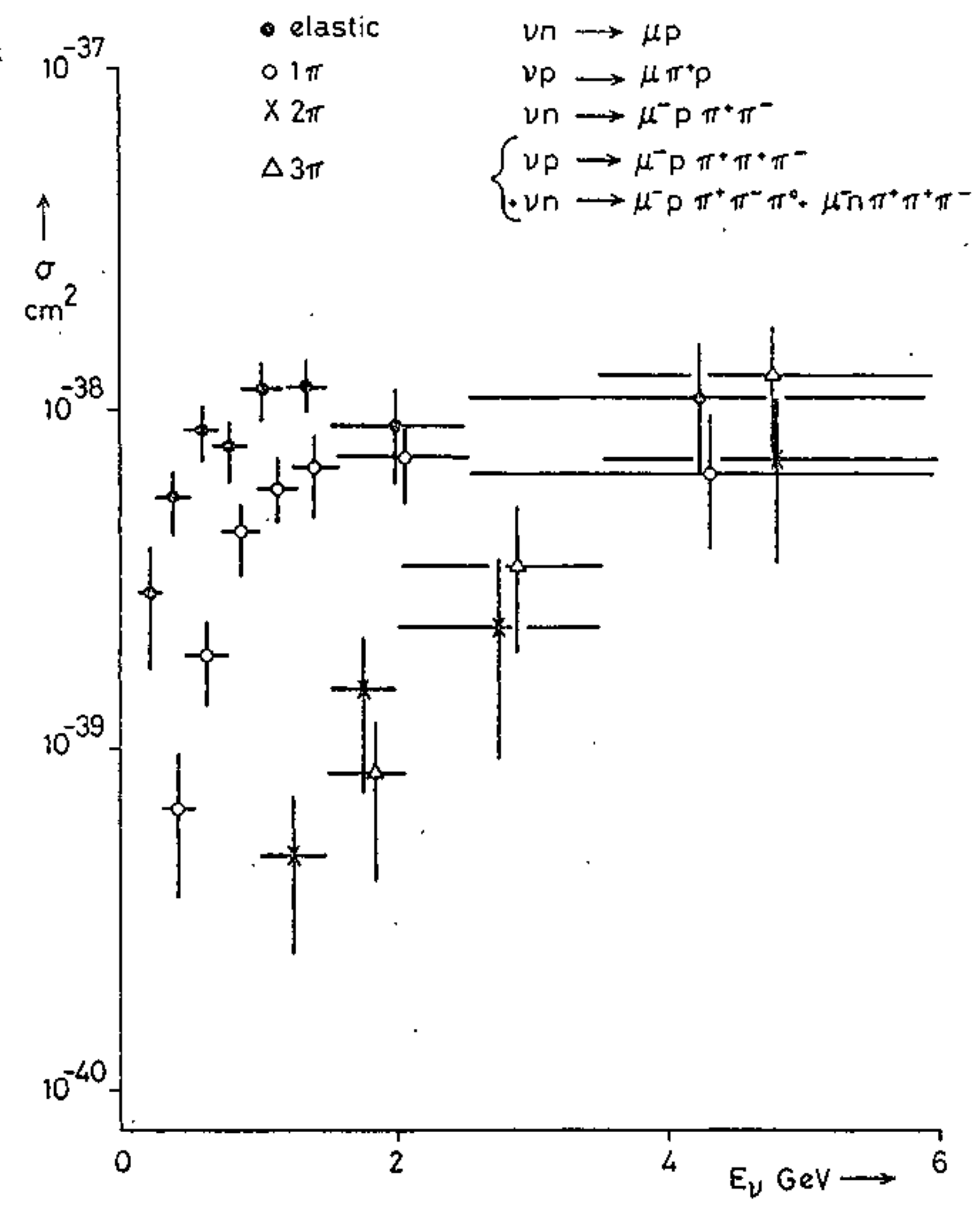

Fig. '3 
$20^{\circ}+60^{6} F_{g} 6$

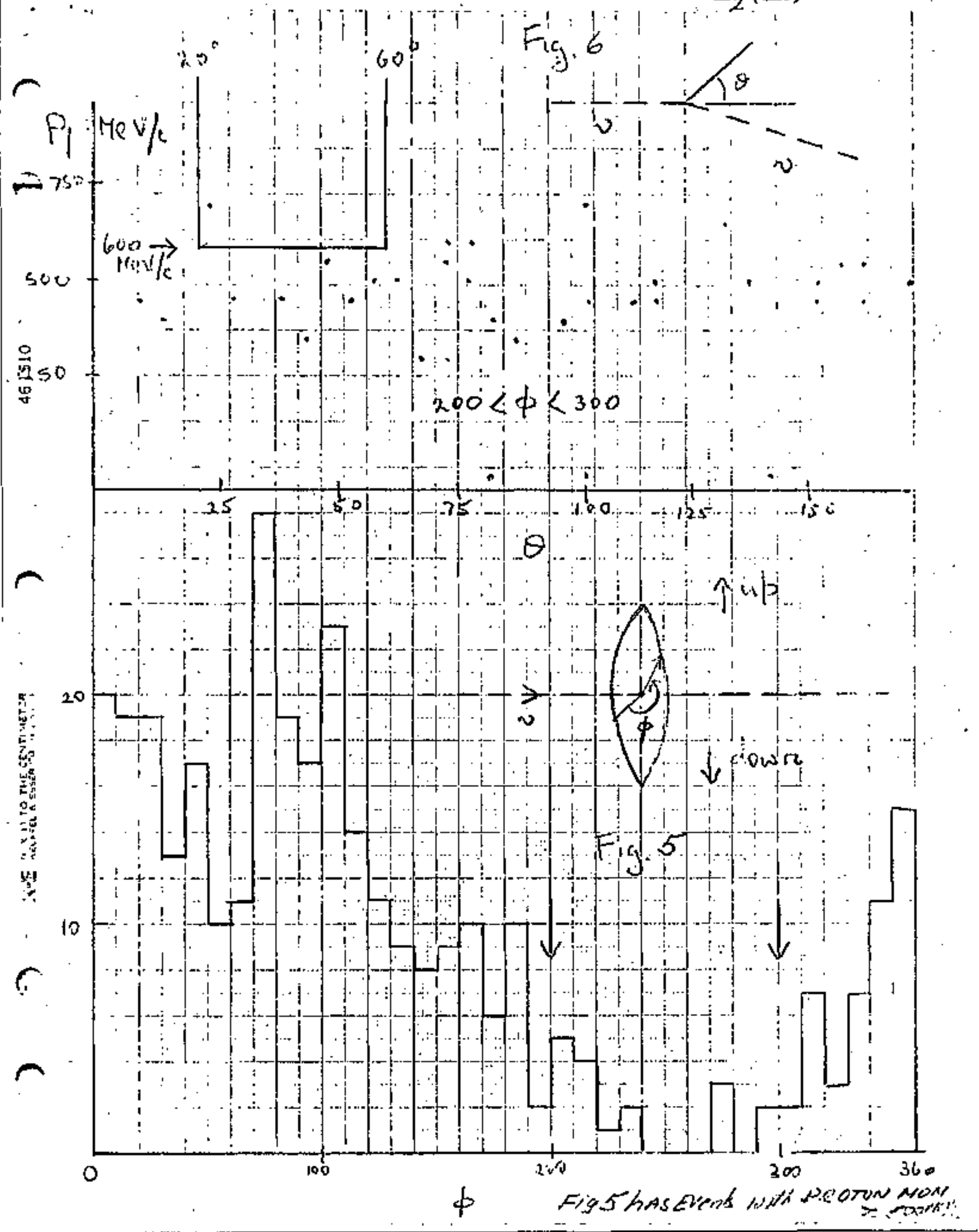




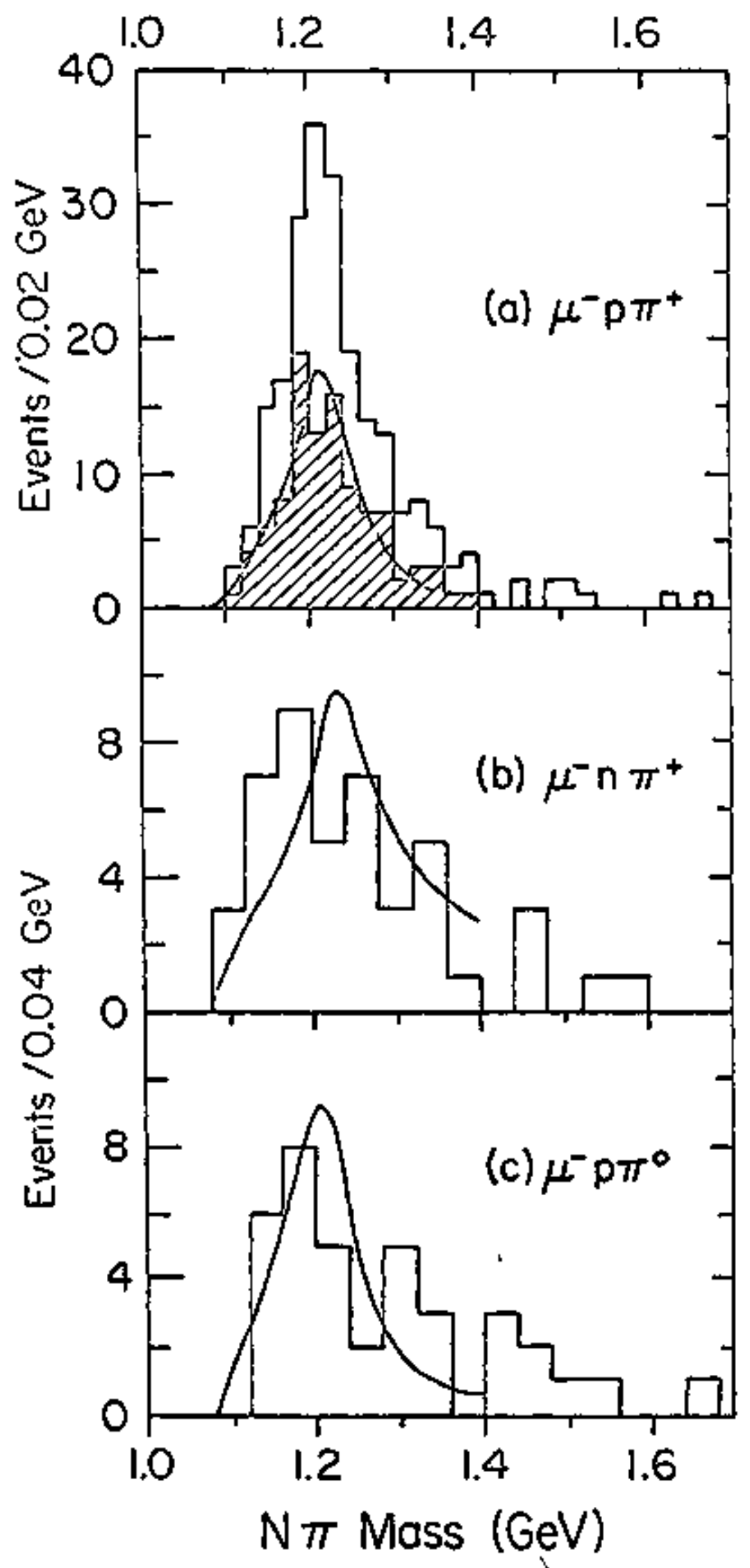

Fig. 8 
$\Gamma$

$\nu n \rightarrow \mu^{-} \rho \pi^{\circ}$

- $A L L \gamma$

- $\gamma$ WHICH HIT PLATES

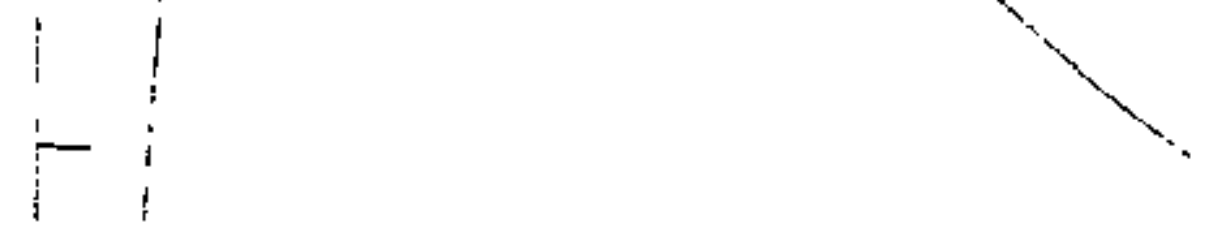

-

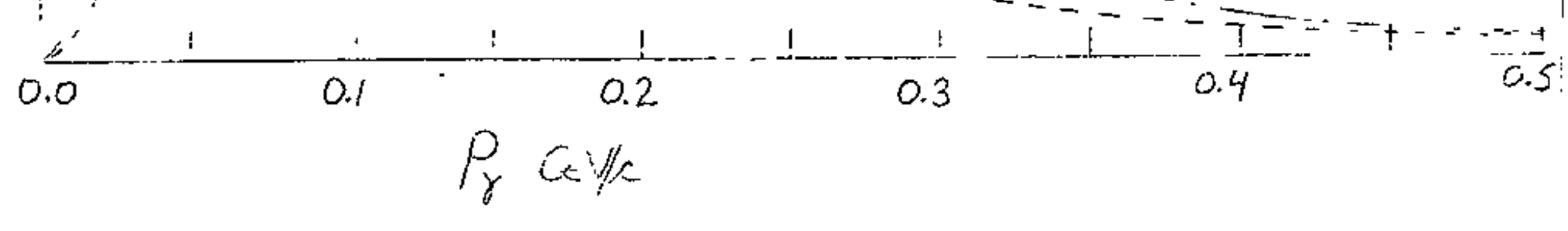

Eng. 9 